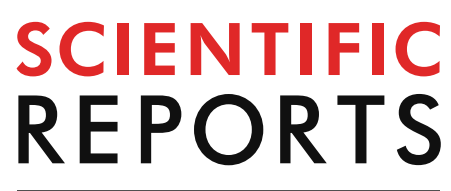

natureresearch

Check for updates

\title{
OPEN TNFR1, TNFR2, neutrophil gelatinase-associated lipocalin and heparin binding protein in identifying sepsis and predicting outcome in an intensive care cohort
}

\author{
Maria Bergquist $^{1 \bowtie}$, Line Samuelsson², Anders Larsson ${ }^{3}$, Jonas Tydén ${ }^{2}$, Joakim Johansson² \& \\ Miklos Lipcsey ${ }^{4}$
}

To date no biomarkers can aid diagnosing sepsis with adequate accuracy. We set out to assess the ability of Tumor necrosis factor receptor (TNFR) 1 and 2, Neutrophil gelatinase-associated lipocalin (NGAL) and Heparin binding protein (HBP) to discriminate sepsis from non-infected critically ill patients in a large ICU cohort, and to evaluate their value to predict mortality at 30 days. Adult patients admitted to the ICU with an arterial catheter were included. Clinical data and blood samples were prospectively recorded daily. Diagnoses were set retrospectively. Descriptive statistics and logistic regression models were used. NGAL, TNFR1 and TNFR2 were higher in sepsis patients compared to other diagnoses, as well as in non-survivors compared to survivors. In addition, these biomarkers increased with increasing stages of acute kidney injury. TNFR1 and TNFR2 performed similarly to NGAL and CRP in identifying sepsis patients, but they performed better than CRP in predicting 30-day mortality in this ICU cohort. Thus, TNFR1 and TNFR2 may be particularly useful in identifying high risk sepsis patients and facilitate relevant health care actions in this group of sepsis patients.

Initiating treatment for sepsis has major impact on outcome ${ }^{1,2}$. However, differentiating sepsis from other causes of critical illness can be challenging. Most biomarkers for diagnosing severe infections measure inflammatory activity that are also triggered by other conditions with systemic inflammation such as burn injuries or after major surgery $^{3,4}$. In the intensive care unit (ICU) where patients with systemic inflammation are common, biomarkers with high specificity for sepsis are of great interest. As the cellular response of the innate immune system to microbial triggers is an early and key event in the inflammatory response of sepsis ${ }^{5}$ biomarkers related to these events could be useful for identifying patients with sepsis.

Tumor necrosis factor (TNF) is a potent pro-inflammatory cytokine with a pivotal signaling role in the host defense against infection and injury, as well as controlling the survival of target cells ${ }^{6}$. It exerts immunological effects through two cell surface receptors, TNFR1 (p55TNFR) and TNFR2 (p75TNFR). TNFR1 is the main mediator of TNF and ubiquitously expressed while TNFR2 is mainly expressed on immune cells ${ }^{7}$. Activation through ligand binding of TNFR1 activates mitogen-activated protein kinase pathways and IkB kinase complex, to initiate apoptosis ${ }^{8}$. In contrast, activation of TNFR2 will mobilize and activate NFkB, a transcription factor complex promoting cell survival and proliferation ${ }^{6}$. Although TNFRs are initially membrane-anchored proteins, previous studies have reported proteolytic shedding of TNF receptors during inflammation and after TNF stimulation $^{9-12}$. TNFR1 shedding has also been observed to precede the secretion of TNF in vitro and in vivo ${ }^{13}$, suggesting that increased circulating levels of TNFR1 may function to neutralize the effects of TNF to dampen

${ }^{1}$ Department of Medical Sciences, Clinical Physiology, Uppsala University, Uppsala, Sweden. ${ }^{2}$ Department of Surgical and Perioperative Sciences, Anaesthesiology and Critical Care Medicine (Östersund), Umeå University, Umeå, Sweden. ${ }^{3}$ Department of Medical Sciences, Clinical Chemistry, Uppsala University Hospital, Uppsala, Sweden. ${ }^{4}$ Hedenstierna Laboratory, CIRRUS, Anaesthesiology and Intensive Care, Department of Surgical Sciences, Anesthesiology, Uppsala University, Uppsala, Sweden. ${ }^{\square}$ email: Maria.bergquist@medsci.uu.se 


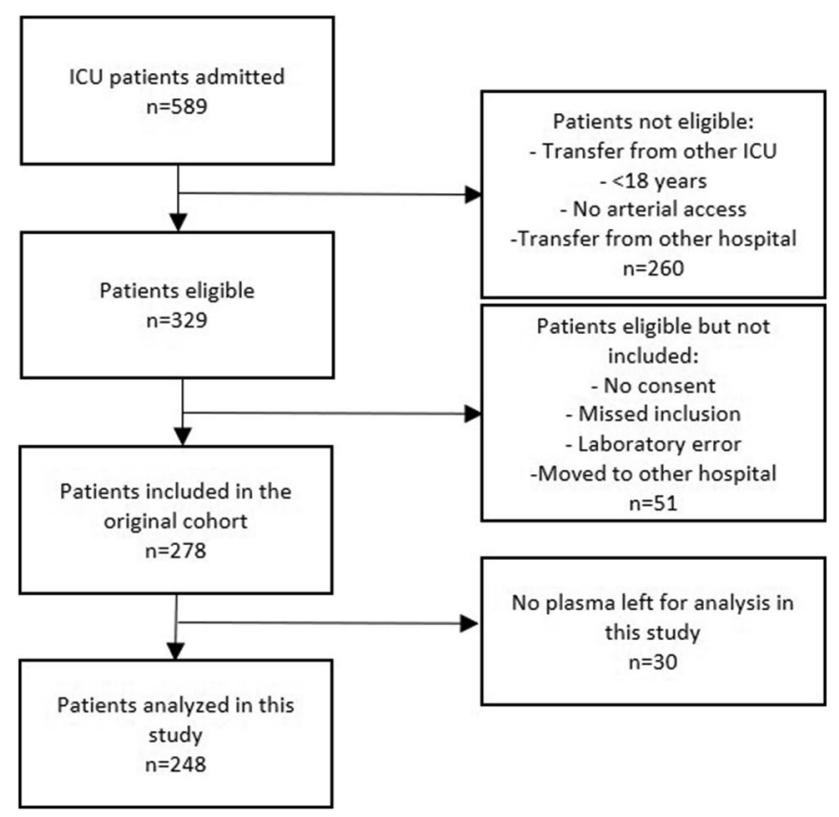

Figure 1. Flow of patients.

an exaggerated host immune response. An increased concentration of circulating TNFRs in combination with a decreased concentration of available cell surface receptor proteins, could result in a significant attenuation of the pro-inflammatory TNF activity and bioavailability. Supporting this hypothesis, defective shedding has been observed to cause innate immune hyperresponsiveness and in vivo toxicity from TNF and LPS ${ }^{13}$. To our knowledge, although TNFRs have been suggested as potential biomarkers of sepsis, they have not previously been evaluated in discriminating sepsis from other inflammatory conditions found in the ICU setting.

Neutrophil gelatinase-associated lipocalin (NGAL) is a critical component of innate immunity to bacterial infection ${ }^{14}$, released by neutrophils and macrophages as well as renal tubular cells ${ }^{15}$, explaining why it is best known as a marker of acute kidney injury $(\mathrm{AKI})^{15}$. It is considered a very early predictor of renal dysfunction as its elevation can be detected 24-36 h prior to changes in serum creatinine. In addition to AKI, NGAL has also been observed to be elevated in coronary artery disease and heart failure, and to be a strong predictor of outcome ${ }^{16,17}$. In recent studies, NGAL concentrations have been associated with inflammatory response, endothelial activation and clinical outcome in sepsis ${ }^{18-20}$. However, it has not been confirmed if increased concentrations of plasma NGAL could be used as an early biomarker to identify patients with sepsis from patients with inflammation rising from other causes than infection.

In several severe inflammatory conditions seen in the ICU setting, vascular leakage constitutes a major clinical challenge $\mathrm{e}^{21}$ and is thought to be driven by proteins released by granulocytes. Heparin Binding Protein (HBP) is released immediately from neutrophil vesicles upon neutrophil activation and besides having anti-microbial and chemotactic characteristics, has also been observed to increase vascular permeability ${ }^{22}$. As both capillary leakage and neutrophil activation are highly dysregulated in patients with sepsis, it is possible that HBP could be detected early during infection and be predictive of outcome and mortality in sepsis. Previous studies have associated HBP levels to circulatory, respiratory and renal failure, as well as to mortality ${ }^{23-27}$. If HBP can be used in discriminating infectious conditions from non-infections inflammation in the ICU remains to be confirmed. Our hypothesis was that TNFR1 and 2, NGAL and HBP could identify patients with sepsis in the ICU.

The objective of this study was to assess the ability of these biomarkers to discriminate sepsis from noninfected critically ill patients in a large ICU cohort, and to evaluate their value to predict mortality at 30 days in this mixed ICU population.

\section{Results}

Out of 589 patients admitted to the intensive care unit, the numbers of eligible and included patients and reasons for exclusions are presented in Fig. 1. Demographics of the cohort and the subgroups of patients with sepsis and no sepsis are presented in Table 1, and of patients with medical events with low inflammation, trauma and other medical conditions in Suppl Table 1. Patient age, SAPS3 score, SOFA score and 30 day mortality was relatively lower in the group of patients with trauma compared to the other diagnosis groups. Mortality at 30 days was highest in the other medical conditions diagnosis group (33\%).

Biomarker concentrations in critically ill patients. The concentrations of plasma NGAL, TNFR1 and TNFR2 in patients with sepsis and non-sepsis on admission are illustrated in Fig. 2a. HBP levels have been reported previously ${ }^{24}$. Biomarker concentrations in the subgroups trauma, medical events with low inflammation and other medical conditions are displayed in Suppl Fig. 1. The highest mean concentrations of these bio- 


\begin{tabular}{|c|c|c|c|}
\hline & All patients $(n=278)$ & Sepsis $(n=89,32 \%)$ & Non-sepsis $(n=189,68 \%)$ \\
\hline Age, years & $68(53-76)$ & $73(62-76)$ & $67(49-75)$ \\
\hline Female sex, n (\%) & $106(38)$ & $35(39)$ & $71(38)$ \\
\hline SAPS3 score & $58(48-70)$ & $65(56-74)$ & $54(46-66)$ \\
\hline Max SOFA & $6(4-10)$ & $8(6-11)$ & $4(2-7)$ \\
\hline ICU LOS, days & $2(1-3)$ & $3(1-6)$ & $2(1-3)$ \\
\hline Mortality at 30 days, n (\%) & $64(23)$ & $18(20)$ & $46(24)$ \\
\hline AKI, n (\%) & $99(35)$ & $44(49)$ & $55(29)$ \\
\hline ARDS, n (\%) & $33(12)$ & $16(18)$ & $13(7)$ \\
\hline \multicolumn{4}{|l|}{ Bacterial foci, $\mathbf{n}(\%)$} \\
\hline Respiratory & & $30(34)$ & \\
\hline Bacteremia with unknown focus & & $18(20)$ & \\
\hline Abdominal & & $16(18)$ & \\
\hline Urogenital & & $10(11)$ & \\
\hline Wound/Soft tissue & & $8(9)$ & \\
\hline CNS & & $6(7)$ & \\
\hline Endocarditis & & $1(1)$ & \\
\hline
\end{tabular}

Table 1. Patient demographics and clinical parameters. Data are presented as median (IQR) unless otherwise stated. SAPS simplified acute physiology score, SOFA sequential organ failure assessment, ICU intensive care unit, $L O S$ length of stay, $A K I$ acute kidney injury, $A R D S$ acute respiratory distress syndrome, $C N S$ central nervous system.
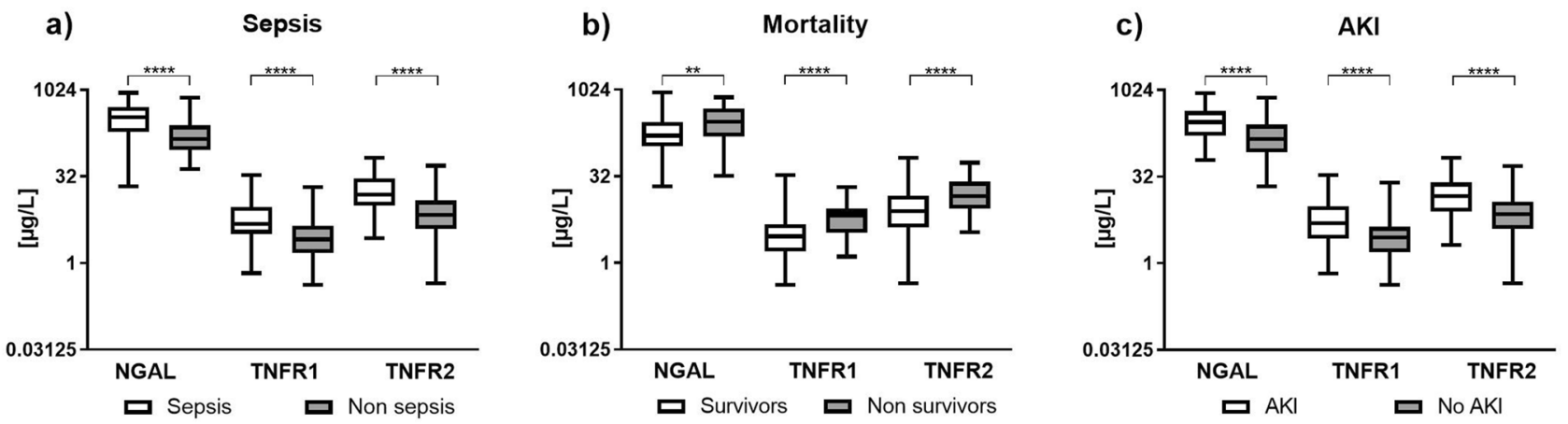

Figure 2. Biomarker concentrations in plasma from patients on admission to the ICU stratified by diagnostic groups; sepsis $(n=89)$ and non-sepsis $(n=189)$, survivors $(n=214)$ and non-survivors $(n=64)$, and presence of acute kidney injury (AKI, $n=99)$ and no AKI $(n=179)$. Box plot summaries of NGAL, TNFR1 and TNFR2 concentration are displayed in natural logarithm scale. Asterisks indicate statistical difference between groups using Mann-Whitney U test. ${ }^{* *} \mathrm{p}<0.01,{ }^{* * * *} \mathrm{p}<0.0001$.

markers were all found in the sepsis group, and this difference was consistent over the three ICU days for TNFR1 and TNFR2 (Fig. 3a).

The performance of biomarkers at cut-off values. The c-indexes for all biomarkers were $>0.7$ for NGAL, TNFR1 and TNFR2 for discriminating patients with sepsis from patients without sepsis (Table 2). In comparing sepsis with all other diagnosis groups, CRP displayed the highest c-index and WBC the lowest. For discriminating sepsis from non-sepsis patients at the identified best cut-off, HBP had highest sensitivity, whereas CRP had the highest specificity, negative predictive value (NPV) and positive predictive value (PPV). For discriminating sepsis vs. medical events with low inflammation and trauma patients at the identified best cut-off, CRP had highest sensitivity, specificity, NPV, and PPV, although TNFR2 demonstrated equally high sensitivity in discriminating sepsis from trauma patients. Combining biomarkers added little improvement to the models in identifying sepsis patients.

When adjusting for age, SOFA score on admission, SAPS3, and ICU LOS, only HBP and WBC were not independent predictors of sepsis when compared to patients in the medical events with low inflammation group.

Biomarker association with renal function. Plasma concentrations of NGAL, TNFR1 and TNFR2 were higher in patients with AKI compared to patients without AKI (Fig. 2c) and did not change over the 

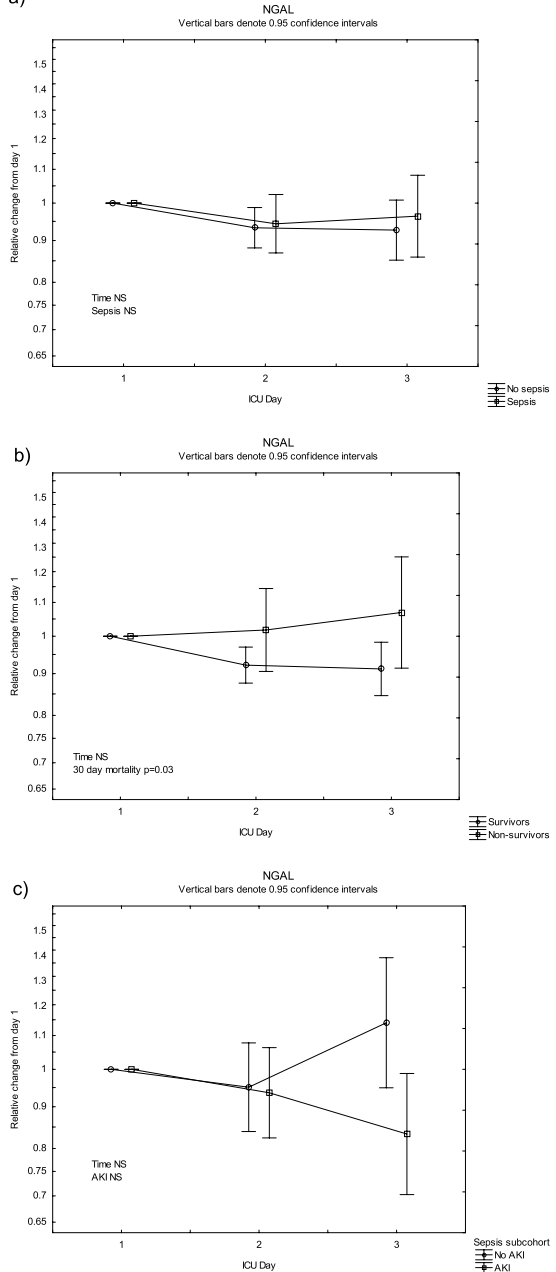
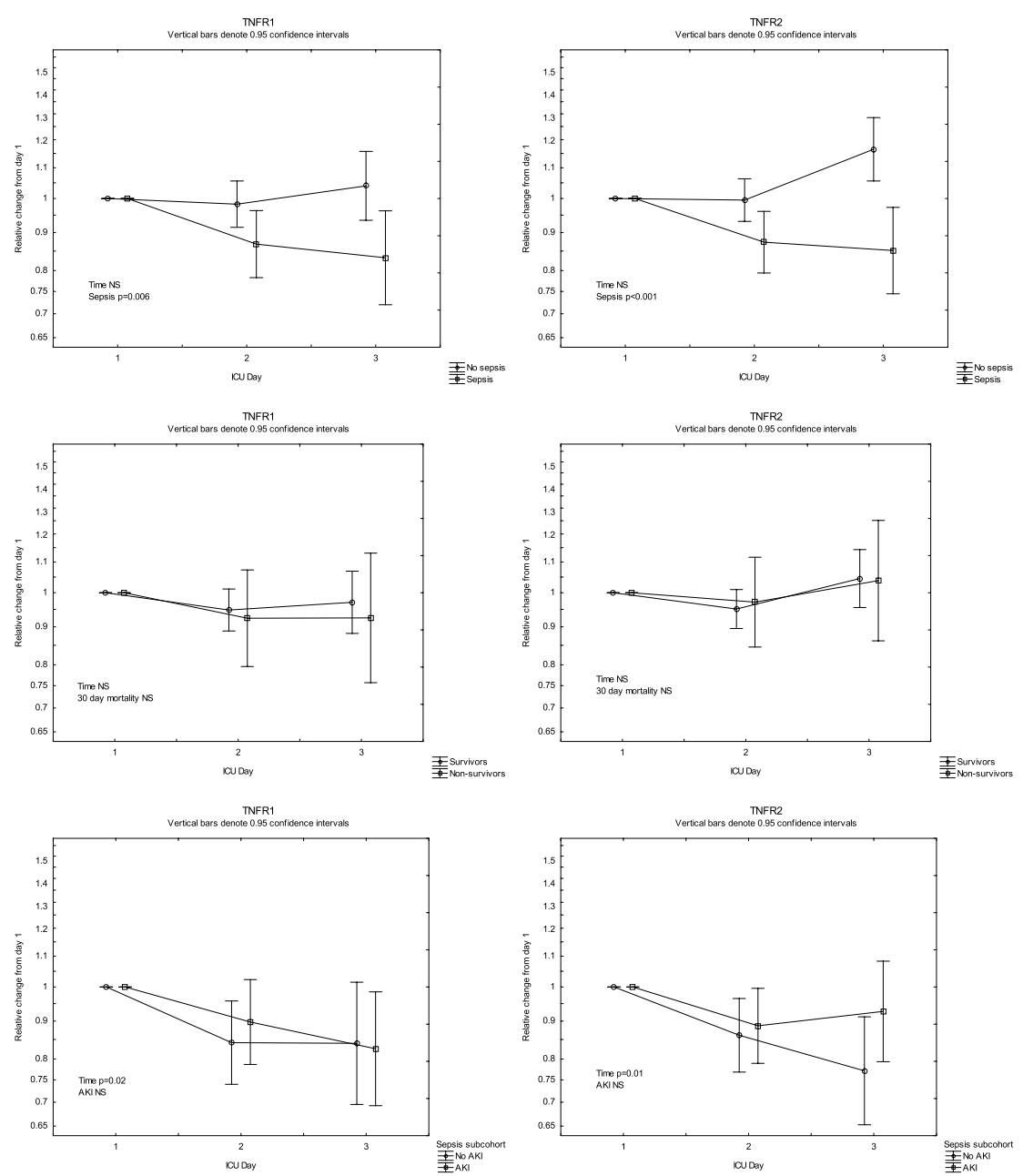

Figure 3. Plasma concentrations NGAL, TNFR1 and TNFR2 from admission (Day 1 ) to Day 3 in the ICU, stratified by (a) sepsis $(n=89)$ and non-sepsis $(n=189),(\mathbf{b})$ survivors $(n=214)$ and non-survivors $(n=64)$, and presence of acute kidney injury (AKI, $n=99)$ and no AKI $(n=179)$. ANOVA III for repeated measures was used to assess differences over time.

three ICU days (Fig. 3b). When stratifying patients according to their AKI stage, NGAL, TNFR1 and TNFR2 increased with increasing level of AKI (Fig. 4).

Biomarker association with outcome. Plasma concentrations of NGAL, TNFR1 and TNFR2 were higher in the non-survivors as compared to survivors as measured on admission to the ICU (Fig. 2b). While TNFR1 and TNFR2 concentrations did not change significantly during the three ICU days, the NGAL concentrations diverged between survivors and non-survivors (Fig. 3c).

The c-indexes for all biomarkers were $>0.6$ for NGAL, TNFR1 and TNFR2 for discriminating survivors from non-survivors (Table 2) with the lowest c-index value for WBC and the highest for TNF1 and TNFR2. For discriminating survivors from non-survivors at the identified best cut-off, HBP had highest sensitivity, TNFR1 the highest specificity. PPV were low for all four biomarkers for mortality at 30 days. NPV were high for all biomarkers with the highest values seen for TNFR1 and TNFR2.

In the univariate regression analysis (Table 3) all biomarkers exhibited significant correlations to age, SOFA score, SAPS3, and ICU LOS apart from HBP vs ICU LOS. NGAL, TNFR1 and TNFR2 vs SOFA score and SAPS3 showed the highest correlation coefficients.

WBC was the only independent predictor of death at 30 days after adjusting for age, SOFA score, SAPS3, and ICU LOS (Table 4).

\section{Discussion}

Our data show that HBP, NGAL, TNFR1 and TNFR2 are higher in sepsis patients compared to other diagnoses, suggesting that these biomarkers could be of value in discriminating patients with infection from other severe inflammatory conditions. Of these biomarkers, TNFR2 performed nominally best in discriminating sepsis patients from non-infected medical patients. With the chosen cut-off values for sepsis in this study, CRP had higher sensitivity and specificity than other biomarkers for identifying sepsis patients in this ICU cohort. 
However, both TNFR1 and TNFR2 performed better than CRP in predicting outcome. WBC was the only independent predictor of mortality at 30 days after adjusting for age and acute and chronic illness severity.

Sepsis is a complex syndrome with systemic inflammation and is still associated with high mortality and its early diagnosis is critical for timely management and patient survival ${ }^{28}$. New clinical and laboratory tools to distinguish between bacterial and non-bacterial conditions are urgently needed to identify patients with severe infections early. Today, there is no available test to diagnose sepsis accurately. Instead, a number of non-specific clinical parameters and the suspicion of infection define sepsis. To confirm suspected infection, several biomarkers are used clinically. The most widely used biomarker is procalcitonin, which has been shown to have some advantages over CRP. Yet procalcitonin has limitations for diagnosis of sepsis and studies on guiding antibiotic therapy with this biomarker have been conflicting regarding patient benefit ${ }^{29,30}$. In the present study we analyzed clinical biomarkers in plasma from a mixed ICU population and found that NGAL and TNFR1 and 2 are associated with poor prognosis and can be used as support in identifying patients with sepsis, especially those with high mortality risk, upon admission to the ICU.

In line with our results, NGAL has previously been associated with increased clinical severity and diagnosis of sepsis $^{19,31,32}$. As NGAL is considered an endogenous bacteriostatic protein expressed to large extent by neutrophils and macrophages, it plays a critical role in the innate immunity against bacterial infection ${ }^{14}$. In contrast to this, it could also hold anti-inflammatory properties by decreasing apoptosis in host immune cells ${ }^{33}$. Taken this into account, it is not surprising that NGAL would increase during sepsis and be of value for the early detection and monitoring of infection. However, identifying patients with sepsis in the ICU is challenging ${ }^{3}$ since activation of an immune response and inflammation is common both with and without infectious etiology ${ }^{29}$. In this population, as shown in the current study, NGAL and CRP are particularly useful for identifying sepsis patients with high specificity and initiating adequate treatment.

Although increased plasma levels of TNFRs in systemic inflammatory conditions have been described decades ago $^{34}$, these soluble receptors of proximal inflammation mediators have not been evaluated as sepsis markers. Although it is a common hypothesis that the shedding of TNF receptors binds and neutralize circulating TNF ${ }^{34}$, it cannot be excluded that TNF receptors act as a reservoir for TNF and thus prolonging its pro-inflammatory action $^{35}$. It is thus not clear if the observed increased shedding of TNFRs in sepsis contribute to a worse outcome or is reflecting an anti-inflammatory response to protect from a deleterious immune reaction to infection. In our study these biomarkers performed with the highest overall combination of sensitivity and specificity for sepsis and were predictors of sepsis even when adjusted for illness severity, age and organ failure. Thus, TNFR1 and 2 could be useful in identifying sepsis patients. Our study does not aim to explain the causal relationship between the increased concentrations of circulating TNFRs and sepsis diagnosis.

Increased HBP levels in sepsis patients in this cohort have been reported previously ${ }^{24}$ and have been investigated as sepsis biomarkers in other critically ill patient populations ${ }^{36}$. Compared to the latter study, HBP had higher c-index in this study for discriminating sepsis patients from other groups. At the set best cut-off, sensitivity was high, negative predictive value was good among the tested biomarkers, but the specificity was low. Thus, sepsis is highly unlikely with low levels HBP in the ICU cohort and using HBP as a biomarker could aid withholding antibiotics in patients without infection.

In this study, $35 \%$ of the patients developed AKI, and $49 \%$ of the patients with sepsis. Our finding that TNFRs correlate with renal function in sepsis is in agreement with previous studies ${ }^{37-39}$. It has been suggested that elevated concentrations of circulating TNFRs could be an effect of impaired renal clearance following organ dysfunction in sepsis ${ }^{37}$. However, the association between increased TNFRs and renal failure remained even in patients with low serum creatinine, suggesting that impaired renal clearance alone cannot explain the increase in soluble TNFRs ${ }^{39}$. In our study, we observed a strong correlation between TNFRs and AKI stage, which to our knowledge has not previously been documented. The finding that concentrations of NGAL, TNFR1 and TNFR2 are higher in patients with AKI compared to patients without AKI, in combination with that these biomarkers are increasing with AKI stage, supports the hypothesis that AKI in this population is driven by inflammation.

The concentrations of these biomarkers were higher in patients who died compared to patients who survived, which is consistent with previous studies ${ }^{40}$. However, in our study we found that they could not be used as independent predictors of mortality after adjusting for illness severity, age and organ failure. This could be explained by the very heterogeneous group of patients with different infecting agents and varying co-morbidities. Moreover, the prediction of mortality with these biomarkers was adjusted for severity of illness, age and the extent of organ failure that together or separately are good or excellent predictors of mortality.

This study, to the best of our knowledge, is the first large prospective investigation of soluble TNFRs in a mixed ICU cohort, comparing sepsis to other non-infectious inflammatory conditions. To facilitate comparison of our data to other studies and clinical practice, we also report the performance of NGAL and HBP which have been investigated previously, as well as two of the most widely used biomarkers CRP and WBC. Moreover, this cohort of patients includes a wide variety of patients admitted to the ICU, which we consider will reflect the performance of these biomarkers more accurately, rather than drawing conclusions from comparisons with healthy controls. As patients were included consecutively, this study could closer reflect how these biomarkers could be utilized in the clinical environment. Finally, the diagnosis of sepsis was made by two independent experts increasing the accuracy of the diagnosis.

Our study has several limitations. Firstly, the analyses were performed post-hoc on an existing material from a previous study. However, the analysis plan was determined prior to commencing this study. Secondly, data on clinical use of antibiotics or immune modulatory drugs (e.g. glucocorticoids) was not available, which may have affected the plasma concentrations of these biomarkers. Apart from studying TNFR1 and 2 in different populations, future studies on guiding antibiotic therapy with TNFRs, NGAL and HBP according to their sensitivity and specificity would be of value. Another limitation is that this study was not designed to collect microbiological data in this ICU cohort. Patients are usually diagnosed with sepsis on clinical grounds, as positive blood cultures 


\begin{tabular}{|c|c|c|c|c|c|c|}
\hline Biomarker levels on admission & C-index & Best cut-off & Sensitivity & Specificity & PPV & NPV \\
\hline \multicolumn{7}{|l|}{ Sepsis vs non-sepsis patients } \\
\hline $\operatorname{HBP}(\mu \mathrm{g} / \mathrm{L})$ & $0.67(0.60-0.74)$ & 30 & 87 & 43 & 41 & 87 \\
\hline NGAL(pg/L) & $0.77(0.71-0.83)$ & 185 & 79 & 69 & 56 & 87 \\
\hline TNFR1 (ng/L) & $0.74(0.67-0.81)$ & 3.0 & 81 & 57 & 48 & 86 \\
\hline TNFR2 (ng/L) & $0.78(0.72-0.84)$ & 9.0 & 81 & 65 & 54 & 87 \\
\hline WBC $\left(\times 10^{9}\right)$ & $0.41(0.34-0.48)$ & - & - & - & - & - \\
\hline $\mathrm{CRP}(\mathrm{mg} / \mathrm{L})$ & $0.83(0.77-0.89)$ & 44 & 86 & 73 & 62 & 91 \\
\hline All biomarkers without WBC & $0.86(0.81-0.91)$ & & 76 & 86 & & \\
\hline \multicolumn{7}{|l|}{ Sepsis vs trauma } \\
\hline $\operatorname{HBP}(\mu \mathrm{g} / \mathrm{L})$ & $0.76(0.66-0.86)$ & 40 & 63 & 74 & 85 & 44 \\
\hline NGAL (pg/L) & $0.86(0.78 .0 .94)$ & 185 & 79 & 84 & 94 & 58 \\
\hline TNFR1 (ng/L) & $0.86(0.78-0.94)$ & 3.4 & 74 & 80 & 92 & 51 \\
\hline TNFR2 (ng/L) & $0.89(0.82-0.96)$ & 6.4 & 90 & 72 & 90 & 72 \\
\hline $\mathrm{WBC}\left(\times 10^{9}\right)$ & $0.46(0.35-0.57)$ & - & - & - & - & - \\
\hline $\mathrm{CRP}(\mathrm{mg} / \mathrm{L})$ & $0.90(0.83-0.97)$ & 27 & 90 & 87 & 95 & 74 \\
\hline All biomarkers without WBC & $0.95(0.90-0.99)$ & & 93 & 86 & & \\
\hline \multicolumn{7}{|c|}{ Sepsis vs medical events with low inflammation } \\
\hline HBP $(\mu \mathrm{g} / \mathrm{L})$ & $0.71(0.63-0.79)$ & 30 & 84 & 55 & 69 & 75 \\
\hline NGAL(pg/L) & $0.81(0.74-0.88)$ & 175 & 80 & 77 & 81 & 77 \\
\hline TNFR1 (ng/L) & $0.77(0.70-0.84)$ & 3.0 & 81 & 62 & 72 & 73 \\
\hline TNFR2 (ng/L) & $0.82(0.75-0.89)$ & 8.7 & 80 & 69 & 72 & 78 \\
\hline WBC $\left(\times 10^{9}\right)$ & $0.44(0.35-0.53)$ & - & - & - & - & - \\
\hline $\mathrm{CRP}(\mathrm{mg} / \mathrm{L})$ & $0.88(0.82-0.94)$ & 44 & 86 & 78 & 84 & 81 \\
\hline All biomarkers without WBC & $0.92(0.87-0.97)$ & & 82 & 95 & & \\
\hline \multicolumn{7}{|l|}{30 day mortality } \\
\hline $\operatorname{HBP}(\mu \mathrm{g} / \mathrm{L})$ & $0.64(0.56-0.72)$ & 39 & 69 & 59 & 33 & 86 \\
\hline NGAL(pg/L) & $0.65(0.57-0.73)$ & 248 & 58 & 69 & 33 & 86 \\
\hline TNFR1 (ng/L) & $0.73(0.65-0.81)$ & 4.8 & 64 & 75 & 40 & 89 \\
\hline TNFR2 (ng/L) & $0.71(0.63-0.79)$ & 12 & 67 & 67 & 34 & 89 \\
\hline WBC $\left(\times 10^{9}\right)$ & $0.59(0.51-0.67)$ & 12.2 & 64 & 54 & 31 & 83 \\
\hline $\mathrm{CRP}(\mathrm{mg} / \mathrm{L})$ & $0.57(0.49-0.65)$ & 39 & 65 & 55 & 30 & 84 \\
\hline All biomarkers & $0.72(0.64-0.80)$ & & 84 & 50 & & \\
\hline
\end{tabular}

Table 2. Performance of biomarkers in discriminating sepsis patients from non-sepsis patients as well as survival. Logistic regression model on biomarkers at admission to intensive care. HBP heparin binding protein, $N G A L$ neutrophil gelatinase-associated lipocalin, TNFR tumor necrosis factor receptor, $W B C$ white blood cell count, CRP C-reactive protein.

in sepsis cohorts are typically around $40 \%{ }^{41}$. However, during the three days after ICU admission, potential community acquired infections are likely to have been captured in this study. Future studies could add further information on the value of using NGAL, TNFR1 and TNFR2 in patients with nosocomial sepsis.

These data suggest that TNFR1 and 2 are promising biomarkers of sepsis in the ICU cohort. The novel biomarkers TNFR1 and TNFR2 performed similarly to NGAL and CRP in identifying sepsis patients. In the same group of patients, high NGAL levels are very suggestive of sepsis, while low HBP levels rule out sepsis. Given its sensitivity and specificity profile, HBP is likely to perform better as a sepsis marker in a cohort with lower incidence of sepsis than the cohort investigated in this study. Additionally, TNFR1 performed better than CRP in predicting 30-day mortality and thus can potentially identify high risk patients with sepsis.

\section{Patients and methods}

Setting. This prospective observational study was performed at the 8-bed mixed ICU of Östersund hospital, Sweden, a 300-bed hospital in Sweden based on material from a previous study ${ }^{24}$.

Data collection and patient groups. All patients admitted to the ICU between 1st of February 2012 and 31st of January 2013 were screened for eligibility. Inclusion criteria were: admission to the ICU and presence of or need for an arterial catheter to be inserted. Patients under the age of 18 years and those transferred from other ICUs were excluded. Clinical data were recorded prospectively daily. Final diagnosis was set retrospectively by review of the patients notes.

Simplified acute physiology score 3 (SAPS 3$)^{42}$ was recorded at admission and Sequential organ failure assessment score (SOFA) ${ }^{43}$ was recorded on admission and then on ICU day 1 and 2 . The last measurement corresponds 

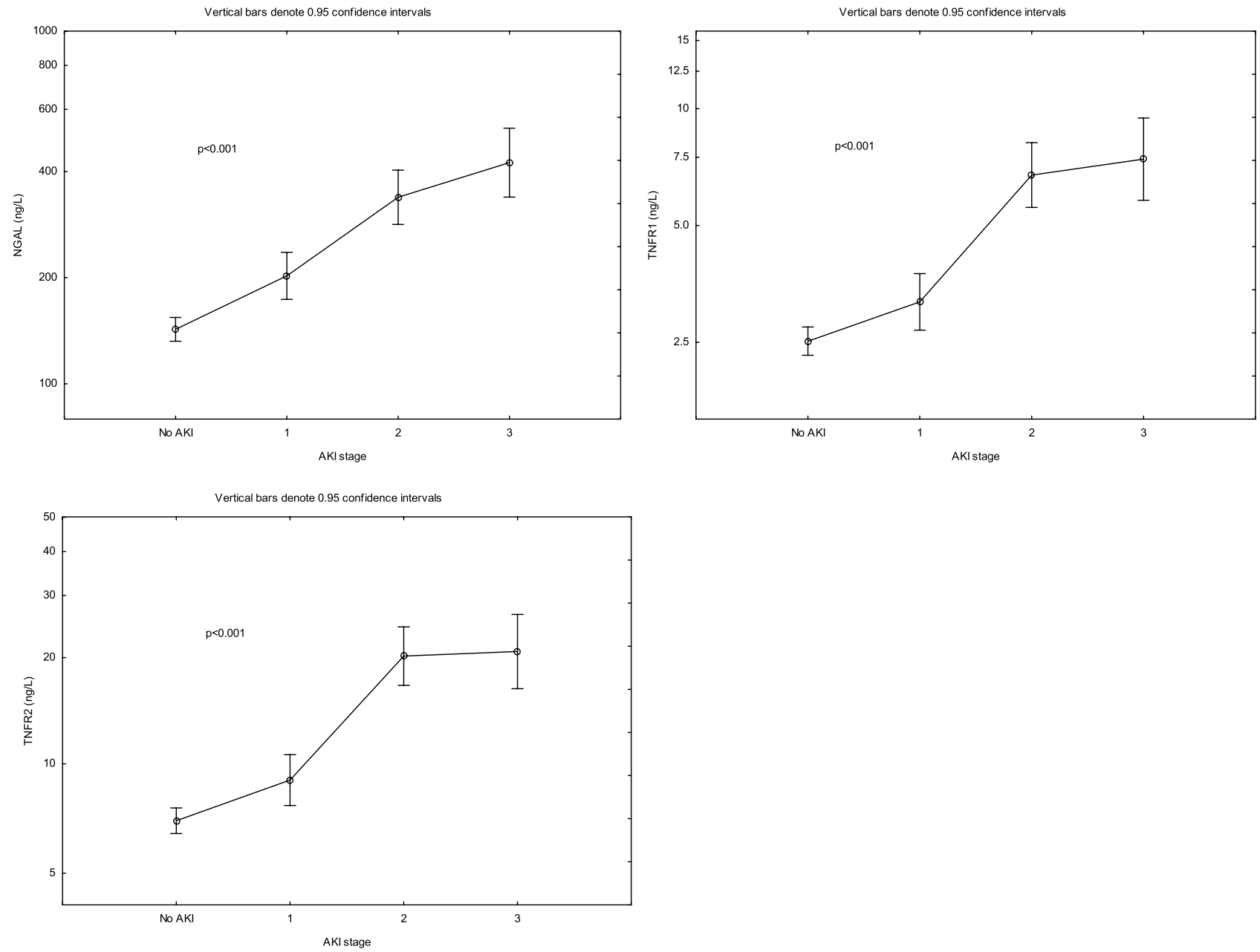

Figure 4. Plasma concentrations NGAL, TNFR1 and TNFR2 in patients stratified by AKI stage. ANOVA III for repeated measures was used to assess group differences.

to a value most often 48-72 $\mathrm{h}$ after admission to the ICU, the exceptions being patients that were discharged, alive or dead, before that registration, where the last available registration was used. Sepsis was defined according to the Sepsis- 3 criteria $^{44}$ with suspected or verified infection and a SOFA on admission of 2 or more. Patients with Acute kidney injury (AKI) were identified ${ }^{45}$.

We predefined four diagnosis groups to compare the performance of the biomarkers in identifying sepsis patients similarly to a previous study of ours $^{46}$ : Sepsis group, according to the definitions above; Trauma group, presumed to have inflammation without infection on admission; Medical events with low inflammation, presumed to be admitted to the ICU without infection; and the remaining patients were designated to the Other medical conditions group.

Handling of samples. Blood samples were collected on admission to the ICU and on the two following days. Routine chemistry including white blood cell count (WBC) and C-reactive protein (CRP) was performed in the hospital central laboratory and registered on the day of admission.

HBP analysis. Concentrations of HPB in plasma were measured as described earlier ${ }^{24}$. Briefly, plates were coated with a mouse monoclonal antibody directed against HBP. Intra-, and inter-assay coefficient of variation were both less than $10 \%$.

NGAL, TNFR1 and TNFR2. Levels of NGAL (DY1757), TNF receptor 1 (DY225) and TNF receptor 2 (DY726) were analyzed using commercially available ELISA kit (R\&D Systems, Minneapolis, MN). The assays had a total coefficient of variation (CV) of approximately $6-7 \%$ and the samples were analyzed as singletons. Laboratory technicians were blinded in relation to patient data.

Statistical analysis. Data are presented as median (IQR) or as number of observations (percent of total number of observations). To compare two or more groups, Mann-Whitney U test or Kruskal Wallis with Dunn's correction were used, respectively. Spearman's correlation test was used for assessing the association between 


\begin{tabular}{|l|l|l|l|l|}
\hline Biomarker & Age (years) & SOFA score & SAPS3 & ICU LOS (DAYS) \\
\hline HBP & rho $=0.21 ; \mathrm{p}=0.0006$ & rho $=0.32 ; \mathrm{p}<0.0001$ & rho $=0.32 ; \mathrm{p}<0.0001$ & NS \\
\hline NGAL & rho $=0.20 ; \mathrm{p}=0.004$ & rho $=0.50 ; \mathrm{p}<0.0001$ & rho $=0.38 ; \mathrm{p}<0.0001$ & rho $=0.17 ; \mathrm{p}=0.01$ \\
\hline TNFr1 & rho $=0.27 ; \mathrm{p}<0.0001$ & rho $=0.49 ; \mathrm{p}<0.0001$ & rho $=0.44 ; \mathrm{p}<0.0001$ & rho $=0.18 ; \mathrm{p}=0.008$ \\
\hline TNFr2 & rho $=0.31 ; \mathrm{p}<0.0001$ & rho $=0.52 ; \mathrm{p}<0.0001$ & rho $=0.47 ; \mathrm{p}<0.0001$ & rho $=0.20 ; \mathrm{p}=0.003$ \\
\hline WBC & NS & NS & NS & NS \\
\hline CRP & rho $=0.15 ; \mathrm{p}=0.02$ & rho $=0.34 ; \mathrm{p}<0.0001$ & rho $=0.36 ; \mathrm{p}<0.0001$ & rho $=0.22 ; \mathrm{p}=0.0009$ \\
\hline
\end{tabular}

Table 3. Biomarker correlations to age and clinical parameters in all patients at admission. Biomarker correlations to age and clinical parameters were calculated using Spearman's correlation test in patients at admission to intensive care. HBP heparin binding protein, $N G A L$ neutrophil gelatinase-associated lipocalin, TNFR tumor necrosis factor receptor, $W B C$ white blood cell count, CRP C-reactive protein, SOFA sequential organ failure assessment, SAPS simplified acute physiology score, ICU Intensive care unit, LOS length of stay. $\mathrm{P}<0.05$ was considered statistically significant.

\begin{tabular}{|c|c|c|c|}
\hline & Odds ratio & 95\% confidence interval & p-value \\
\hline \multicolumn{4}{|c|}{ Sepsis vs non-sepsis patients } \\
\hline $\operatorname{HBP}(\mu \mathrm{g} / \mathrm{L})$ & 1.00 & $(1.00-1.00)$ & NS \\
\hline NGAL(pg/L) & 1.005 & $(1.00-1.01)$ & 0.0001 \\
\hline NGAL adjusted for AKI & 1.00 & $(1.00-1.01)$ & $<0.0001$ \\
\hline TNFR1 (ng/L) & 1.18 & $(1.07-1.30)$ & 0.0008 \\
\hline TNFR2 (ng/L) & 1.08 & $(1.04-1.12)$ & $<0.0001$ \\
\hline $\mathrm{WBC}\left(\times 10^{9}\right)$ & 1.03 & $(0.99-1.08)$ & NS \\
\hline $\mathrm{CRP}(\mathrm{mg} / \mathrm{L})$ & 1.008 & $(1.00-1.01)$ & $<0.0001$ \\
\hline \multicolumn{4}{|l|}{ Sepsis vs trauma } \\
\hline $\operatorname{HBP}(\mu \mathrm{g} / \mathrm{L})$ & 1.03 & $(1.00-1.05)$ & 0.03 \\
\hline NGAL(pg/L) & 1.01 & $(1.00-1.02)$ & 0.002 \\
\hline NGAL adjusted for AKI & 1.02 & $(1.01-1.03)$ & 0.003 \\
\hline TNFR1 (ng/L) & 1.70 & $(1.13-2.56)$ & 0.01 \\
\hline TNFR2 (ng/L) & 1.20 & $(1.03-1.38)$ & 0.01 \\
\hline $\mathrm{WBC}\left(\times 10^{9}\right)$ & 1.00 & $(0.92-1.07)$ & NS \\
\hline $\mathrm{CRP}(\mathrm{mg} / \mathrm{L})$ & 1.02 & $(1.01-1-03)$ & 0.003 \\
\hline \multicolumn{4}{|c|}{ Sepsis vs medical events with low inflammation } \\
\hline $\operatorname{HBP}(\mu \mathrm{g} / \mathrm{L})$ & 1.00 & $(1.00-1.01)$ & NS \\
\hline NGAL(pg/L) & 1.01 & $(1.00-1.01)$ & $<0.0001$ \\
\hline NGAL adjusted for AKI & 1.01 & $(1.00-1.01)$ & $<0.0001$ \\
\hline TNFR1 (ng/L) & 1.29 & $(1.09-1.52)$ & 0.003 \\
\hline TNFR2 (ng/L) & 1.11 & $(1.04-1.19)$ & 0.002 \\
\hline WBC $\left(\times 10^{9}\right)$ & 0.97 & $(0.92-1.02)$ & NS \\
\hline $\mathrm{CRP}(\mathrm{mg} / \mathrm{L})$ & 1.02 & $(1.01-1.03)$ & $<0.0001$ \\
\hline \multicolumn{4}{|l|}{30 day mortality } \\
\hline $\mathrm{HBP}(\mu \mathrm{g} / \mathrm{L})$ & 1.00 & $(1.00-1.00)$ & NS \\
\hline NGAL(pg/L) & 1.00 & $(1.00-1.00)$ & NS \\
\hline NGAL adjusted for AKI & 1.00 & $(1.00-1.00)$ & NS \\
\hline TNFR1 (ng/L) & 0.99 & $(0.93-1.07)$ & NS \\
\hline TNFR2 (ng/L) & 1.00 & $(0.97-1.03)$ & NS \\
\hline WBC $\left(\times 10^{9}\right)$ & 1.06 & $(1.01-1.11)$ & 0.02 \\
\hline $\mathrm{CRP}(\mathrm{mg} / \mathrm{L})$ & 1.00 & $(0.99-1.00)$ & NS \\
\hline
\end{tabular}

Table 4. Odds rations for increases in biomarkers in patients with sepsis vs trauma, sepsis vs other medical conditions, as well as for patients being alive at 30 days after ICU admission, adjusted for sex, SAPS3, maximal ICU SOFA score and age. Logistic regression model on biomarkers at admission to intensive care. $H B P$ heparin binding protein, NGAL neutrophil gelatinase-associated lipocalin, TNFR tumor necrosis factor receptor, WBC white blood cell count, $C R P$ C-reactive protein, SOFA sequential organ failure assessment, SAPS simplified acute physiology score, $I C U$ Intensive care unit. $P<0.05$ was considered statistically significant. 
two variables. We used ANOVA III for repeated measures to assess differences over time. Logistic regression was performed with diagnosis of Sepsis vs. Trauma and Sepsis vs. Other medical conditions, and 30-day mortality as dependent variables with biomarkers as sole predictors to calculate area under the curve of the receiver operating characteristic (c-index). The best cut-off was defined as maximal distance from the diagonal and receiver operating characteristic curve. In the same model after adjusting for age, sex, extent of organ failure (SOFA) and illness severity (SAPS3), we also assessed if the biomarker in the models was an independent predictor of sepsis or death and the magnitude of such an effect expressed as odds ratios. For calculations and figures, STATISTICA software, version 13.2 (StatSoft, Tulsa, OK) and GraphPad Prism 7.0 for Windows (GraphPad Software Inc, La Jolla, CA, USA) were used. $\mathrm{p}<0.05$ was considered significant where relevant.

Ethical approval. The study was approved by the Regional Ethical Review Board (EPN) in Linkoping (No. 2018/16-32). Informed consent was obtained from the patient, or next of kin if the patient was unable give consent. All the experiments were performed in accordance with the relevant guidelines and regulations.

STROBE guidelines were followed for reporting.

\section{Data availability}

The datasets used and/or analyzed during the current study are available from the corresponding author on reasonable request.

Received: 10 February 2020; Accepted: 17 August 2020

Published online: 18 September 2020

\section{References}

1. Seymour, C. W. et al. Time to treatment and mortality during mandated emergency care for sepsis. N. Engl. J. Med. 376, 2235-2244. https://doi.org/10.1056/NEJMoa1703058 (2017).

2. Vester-Andersen, M., Lundstrom, L. H., Buck, D. L. \& Moller, M. H. Association between surgical delay and survival in highrisk emergency abdominal surgery: a population-based Danish cohort study. Scand. J. Gastroenterol. 51, 121-128. https://doi. org/10.3109/00365521.2015.1066422 (2016).

3. Adamina, M. et al. Meta-analysis of the predictive value of C-reactive protein for infectious complications in abdominal surgery. Br. J. Surg. 102, 590-598. https://doi.org/10.1002/bjs.9756 (2015).

4. Stanojcic, M., Vinaik, R. \& Jeschke, M. G. Status and challenges of predicting and diagnosing sepsis in burn patients. Surg. Infect. (Larchmt) 19, 168-175. https://doi.org/10.1089/sur.2017.288 (2018).

5. Rivera, A., Siracusa, M. C., Yap, G. S. \& Gause, W. C. Innate cell communication kick-starts pathogen-specific immunity. Nat. Immunol. 17, 356-363. https://doi.org/10.1038/ni.3375 (2016).

6. Croft, M. The role of TNF superfamily members in T-cell function and diseases. Nat. Rev. Immunol. 9, 271-285. https://doi. org/10.1038/nri2526 (2009).

7. Pimentel-Muinos, F. X. \& Seed, B. Regulated commitment of TNF receptor signaling: a molecular switch for death or activation. Immunity 11, 783-793 (1999).

8. Wajant, H., Pfizenmaier, K. \& Scheurich, P. Tumor necrosis factor signaling. Cell Death Differ 10, 45-65. https://doi.org/10.1038/ sj.cdd.4401189 (2003).

9. Deng, M., Loughran, P. A., Zhang, L., Scott, M. J. \& Billiar, T. R. Shedding of the tumor necrosis factor (TNF) receptor from the surface of hepatocytes during sepsis limits inflammation through cGMP signaling. Sci. Signal 8, 11. https://doi.org/10.1126/scisi gnal.2005548 (2015)

10. Pinckard, J. K., Sheehan, K. C., Arthur, C. D. \& Schreiber, R. D. Constitutive shedding of both p55 and p75 murine TNF receptors in vivo. J. Immunol. 158, 3869-3873 (1997).

11. Diez-Ruiz, A. et al. Soluble receptors for tumour necrosis factor in clinical laboratory diagnosis. Eur. J. Haematol. 54, 1-8 (1995).

12. Lantz, M., Malik, S., Slevin, M. L. \& Olsson, I. Infusion of tumor necrosis factor (TNF) causes an increase in circulating TNFbinding protein in humans. Cytokine 2, 402-406 (1990).

13. Giai, C. et al. Shedding of tumor necrosis factor receptor 1 induced by protein A decreases tumor necrosis factor alpha availability and inflammation during systemic Staphylococcus aureus infection. Infect. Immun. 81, 4200-4207. https://doi.org/10.1128/ IAI.00593-13 (2013).

14. Goetz, D. H. et al. The neutrophil lipocalin NGAL is a bacteriostatic agent that interferes with siderophore-mediated iron acquisition. Mol. Cell. 10, 1033-1043 (2002).

15. Schmidt-Ott, K. M. et al. Dual action of neutrophil gelatinase-associated lipocalin. J. Am. Soc. Nephrol. 18, 407-413. https://doi. org/10.1681/ASN.2006080882 (2007).

16. Lindberg, S. et al. Prognostic utility of neutrophil gelatinase-associated lipocalin in predicting mortality and cardiovascular events in patients with ST-segment elevation myocardial infarction treated with primary percutaneous coronary intervention. J. Am. Coll. Cardiol. 60, 339-345. https://doi.org/10.1016/j.jacc.2012.04.017 (2012).

17. Damman, K., van Veldhuisen, D. J., Navis, G., Voors, A. A. \& Hillege, H. L. Urinary neutrophil gelatinase associated lipocalin (NGAL), a marker of tubular damage, is increased in patients with chronic heart failure. Eur. J. Heart. Fail. 10, 997-1000. https:// doi.org/10.1016/j.ejheart.2008.07.001 (2008).

18. Macdonald, S. P. J. et al. Resistin and NGAL are associated with inflammatory response, endothelial activation and clinical outcomes in sepsis. Inflamm. Res. 66, 611-619. https://doi.org/10.1007/s00011-017-1043-5 (2017).

19. Wang, B. et al. Neutrophil gelatinase-associated lipocalin predicts myocardial dysfunction and mortality in severe sepsis and septic shock. Int. J. Cardiol. 227, 589-594. https://doi.org/10.1016/j.ijcard.2016.10.096 (2017).

20. Chang, W. et al. Predictive utilities of neutrophil gelatinase-associated lipocalin (NGAL) in severe sepsis. Clin. Chim. Acta 481, 200-206. https://doi.org/10.1016/j.cca.2018.03.020 (2018).

21. Marx, G. Fluid therapy in sepsis with capillary leakage. Eur. J. Anaesthesiol. 20, 429-442 (2003).

22. Gautam, N. et al. Heparin-binding protein (HBP/CAP37): a missing link in neutrophil-evoked alteration of vascular permeability. Nat. Med. 7, 1123-1127. https://doi.org/10.1038/nm1001-1123 (2001).

23. Linder, A., Christensson, B., Herwald, H., Bjorck, L. \& Akesson, P. Heparin-binding protein: an early marker of circulatory failure in sepsis. Clin. Infect. Dis. 49, 1044-1050. https://doi.org/10.1086/605563 (2009).

24. Tyden, J., Herwald, H., Sjoberg, F. \& Johansson, J. Increased plasma levels of heparin-binding protein on admission to intensive care are associated with respiratory and circulatory failure. PLoS ONE 11, e0152035. https://doi.org/10.1371/journal.pone.01520 35 (2016). 
25. Johansson, J. et al. Heparin-binding protein (HBP): an early marker of respiratory failure after trauma?. Acta Anaesthesiol. Scand. 57, 580-586. https://doi.org/10.1111/aas.12070 (2013).

26. Chew, M. S. et al. Increased plasma levels of heparin-binding protein in patients with shock: a prospective, cohort study. Inflamm. Res. 61, 375-379. https://doi.org/10.1007/s00011-011-0422-6 (2012).

27. Bentzer, P. et al. Heparin-binding protein is important for vascular leak in sepsis. Intensive Care Med. Exp. 4, 33. https://doi. org/10.1186/s40635-016-0104-3 (2016).

28. Rhodes, A. et al. Surviving sepsis campaign: international guidelines for management of sepsis and septic shock: 2016. Intensive Care Med 43, 304-377. https://doi.org/10.1007/s00134-017-4683-6 (2017)

29. Kibe, S., Adams, K. \& Barlow, G. Diagnostic and prognostic biomarkers of sepsis in critical care. J. Antimicrob. Chemother. 66(Suppl 2), 33-40. https://doi.org/10.1093/jac/dkq523 (2011).

30. Jensen, J. U. et al. Procalcitonin-guided interventions against infections to increase early appropriate antibiotics and improve survival in the intensive care unit: a randomized trial. Crit. Care Med. 39, 2048-2058. https://doi.org/10.1097/CCM.0b013e3182 1 e8791 (2011).

31. Martensson, J. et al. Neutrophil gelatinase-associated lipocalin in adult septic patients with and without acute kidney injury. Intensive Care Med. 36, 1333-1340. https://doi.org/10.1007/s00134-010-1887-4 (2010).

32. Wang, M., Zhang, Q., Zhao, X., Dong, G. \& Li, C. Diagnostic and prognostic value of neutrophil gelatinase-associated lipocalin, matrix metalloproteinase-9, and tissue inhibitor of matrix metalloproteinases-1 for sepsis in the Emergency Department: an observational study. Crit. Care 18, 634. https://doi.org/10.1186/s13054-014-0634-6 (2014).

33. Srinivasan, G. et al. Lipocalin 2 deficiency dysregulates iron homeostasis and exacerbates endotoxin-induced sepsis. J. Immunol. 189, 1911-1919. https://doi.org/10.4049/jimmunol.1200892 (2012).

34. Van Zee, K. J. et al. Tumor necrosis factor soluble receptors circulate during experimental and clinical inflammation and can protect against excessive tumor necrosis factor alpha in vitro and in vivo. Proc. Natl. Acad. Sci. USA 89, 4845-4849 (1992).

35. Aderka, D., Engelmann, H., Maor, Y., Brakebusch, C. \& Wallach, D. Stabilization of the bioactivity of tumor necrosis factor by its soluble receptors. J. Exp. Med. 175, 323-329 (1992).

36. Llewelyn, M. J. et al. Sepsis biomarkers in unselected patients on admission to intensive or high-dependency care. Crit. Care 17, R60. https://doi.org/10.1186/cc12588 (2013).

37. de Werra, I. et al. Cytokines, nitrite/nitrate, soluble tumor necrosis factor receptors, and procalcitonin concentrations: comparisons in patients with septic shock, cardiogenic shock, and bacterial pneumonia. Crit. Care Med. 25, 607-613. https://doi. org/10.1097/00003246-199704000-00009 (1997).

38. Froon, A. H., Bemelmans, M. H., Greve, J. W., van der Linden, C. J. \& Buurman, W. A. Increased plasma concentrations of soluble tumor necrosis factor receptors in sepsis syndrome: correlation with plasma creatinine values. Crit. Care Med. 22, 803-809. https ://doi.org/10.1097/00003246-199405000-00015 (1994).

39. Iglesias, J., Marik, P. E. \& Levine, J. S. Elevated serum levels of the type I and type II receptors for tumor necrosis factor-alpha as predictive factors for ARF in patients with septic shock. Am. J. Kidney Dis. 41, 62-75. https://doi.org/10.1053/ajkd.2003.50024 (2003).

40. Gordon, A. C. et al. TNF and TNFR polymorphisms in severe sepsis and septic shock: a prospective multicentre study. Genes Immun. 5, 631-640. https://doi.org/10.1038/sj.gene.6364136 (2004).

41. Nannan Panday, R. S., Lammers, E. M. J., Alam, N. \& Nanayakkara, P. W. B. An overview of positive cultures and clinical outcomes in septic patients: a sub-analysis of the Prehospital Antibiotics Against Sepsis (PHANTASi) trial. Crit. Care 23, 182. https://doi. org/10.1186/s13054-019-2431-8 (2019).

42. Moreno, R. P. et al. SAPS 3: from evaluation of the patient to evaluation of the intensive care unit: part 2: development of a prognostic model for hospital mortality at ICU admission. Intensive Care Med. 31, 1345-1355. https://doi.org/10.1007/s00134-005-2763-5 (2005).

43. Vincent, J. L. et al. The SOFA (sepsis-related organ failure assessment) score to describe organ dysfunction/failure: on behalf of the working group on sepsis-related problems of the European society of intensive care medicine. Intensive Care Med. 22, 707-710 (1996).

44. Singer, M. et al. The third international consensus definitions for sepsis and septic shock (Sepsis-3). JAMA 315, 801-810. https:// doi.org/10.1001/jama.2016.0287 (2016).

45. Abosaif, N. Y., Tolba, Y. A., Heap, M., Russell, J. \& El Nahas, A. M. The outcome of acute renal failure in the intensive care unit according to RIFLE: model application, sensitivity, and predictability. Am. J. Kidney Dis. 46, 1038-1048. https://doi.org/10.1053/j. ajkd.2005.08.033 (2005).

46. Simm, M. et al. Performance of plasma calprotectin as a biomarker of early sepsis: a pilot study. Biomark. Med. 10, 811-818. https ://doi.org/10.2217/bmm-2016-0032 (2016).

\section{Acknowledgements}

We thank Dr Mats Eriksson for reading the manuscript.

\section{Author contributions}

All authors (M.B., L.S., A.L., J.T., J.J., M.L.) conceived the study and contributed to the design of the study. A.L. preformed the laboratory analysis. M.B. and M.L. did the calculations, analyzed the data and drafted the manuscript. All authors (M.B., L.S., A.L., J.T., J.J., M.L.) contributed to the manuscript and approved the final version.

\section{Funding}

This study was financed by institutional grants from the Uppsala university hospital. Open Access funding provided by Uppsala University.

\section{Competing interests}

The authors declare no competing interests.

\section{Additional information}

Supplementary information is available for this paper at https://doi.org/10.1038/s41598-020-72003-9.

Correspondence and requests for materials should be addressed to M.B.

Reprints and permissions information is available at www.nature.com/reprints.

Publisher's note Springer Nature remains neutral with regard to jurisdictional claims in published maps and institutional affiliations. 
(c) (i) Open Access This article is licensed under a Creative Commons Attribution 4.0 International cc) License, which permits use, sharing, adaptation, distribution and reproduction in any medium or format, as long as you give appropriate credit to the original author(s) and the source, provide a link to the Creative Commons licence, and indicate if changes were made. The images or other third party material in this article are included in the article's Creative Commons licence, unless indicated otherwise in a credit line to the material. If material is not included in the article's Creative Commons licence and your intended use is not permitted by statutory regulation or exceeds the permitted use, you will need to obtain permission directly from the copyright holder. To view a copy of this licence, visit http://creativecommons.org/licenses/by/4.0/.

(C) The Author(s) 2020 